\title{
Avaliação da permeabilidade transiente de rochas a partir do uso do equipamento AutoLab 1000
}

\author{
Darby Pereira Dantas de Lima1', Arthu
'Universidade de Brasília - UnB/IGD \\ Copyright 2019, SBGf - Sociedade Brasileira de Geofísica \\ This paper was prepared for presentation during the $16^{\text {th }}$ International Congress of the \\ Brazilian Geophysical Society held in Rio de Janeiro, Brazil, 19-22 August 2019. \\ Contents of this paper were reviewed by the Technical Committee of the $16^{\text {th }}$ \\ International Congress of the Brazilian Geophysical Society and do not necessarily \\ represent any position of the SBGf, its officers or members. Electronic reproduction or \\ storage of any part of this paper for commercial purposes without the written consent \\ of the Brazilian Geophysical Society is prohibited.
}

\section{Abstract}

We present some results concerning permeability measurements of two types of rock, sandstone and granite with AutoLab1000. The acquisition of data with complex transients as ASPIKE, Multipulse and SINE6 is useful when comparing the response of permeability in respect of confining pressure and pore pressure to evaluate the rock behavior. The samples of rock for this study are Berea Sandstone and Barre Granite, both from United States of America. Transient permeability is a technique developed at New England Research - NER that measures fluid permeability at in situ conditions applying different values for confining pressure and pore pressure. This means that the equipment controls transient in pore pressure at the upstream side of the sample while monitoring the pore pressure response at the downstream end. After, analytical solutions are applied to fit the response of permeability.

\section{Introdução}

Este trabalho tem como principal objetivo apresentar resultados a partir do equipamento AutoLab 1000 na análise de permeabilidade transiente das rochas. $O$ procedimento consiste na identificação e caracterização da pressão de poro das rochas a partir de variações efetivas das pressões confinantes e aplicadas. Foram utilizados dois tipos de rochas, arenito e granito, que apresentam diferentes porosidades e consequentemente resultados distintos de permeabilidade. As informações de permeabilidade obtidas nos experimentos a partir do AutoLab 1000, com base na estimativa da pressão de poro, possibilitam maior conhecimento das propriedades do espaço poroso e são indicadores do potencial das rochas como aquíferos ou reservatórios, informações importantes para a indústria de petróleo e gás.

O AutoLab 1000 é um sistema servo-hidráulico hidrostático para medidas de permeabilidade, e outras propriedades físicas em amostras de rochas de até 50 mm (2,0 in) de diâmetro em sobrecarga de pressão, pressão de poro e temperatura. O método de medição envolve o controle de transientes complexos na pressão de poro na parte superior amostra (upstream) enquanto monitora a resposta na extremidade inferior (downstream). Tanto o método quanto a técnica de medição foram desenvolvidos pela empresa New England Research - NER. A permeabilidade é calculada ao ajustar a resposta com soluções analíticas, para amostras de alta e baixa permeabilidade em milidarcies $(\mathrm{mD})$, nanodarcies $(\mathrm{nD})$ e microdarcies $(\mu \mathrm{D})$.

O objetivo principal é a comparação da resposta de permeabilidade com o uso de três transientes complexos ASPIKE, Multipulse e SINE6 em pressões distintas. Neste trabalho, os experimentos foram avaliados por dois modos: Alta Permeabilidade (High Perm) ou Baixa Permeabilidade (Low Perm). Em amostras analisadas com Alta Permeabilidade, foram utilizadas pressões confinantes máximas de $60 \mathrm{MPa}$ e pressões de poro máxima de $7 \mathrm{MPa}$. Para amostras avaliadas por Baixa Permeabilidade, as pressões máximas foram de $10 \mathrm{MPa}$ e $5 \mathrm{Mpa}$, respectivamente, para as pressões confinante e de poro.

\section{Materiais}

As amostras utilizadas nos experimentos foram em plugs de rochas com diâmetro e altura de $25 \mathrm{~mm}$ (1,0 in). As amostras de rochas selecionadas apresentam processos de formação distintos onde as principais propriedades do espaço poroso avaliadas foram porosidade $e$ permeabilidade, como: i) rocha sedimentar terrígena com porosidade primária, arenito (Berea Formation, USA) e ii) rochas graníticas com porosidade secundária e granulação média, Barre Granite (USA).

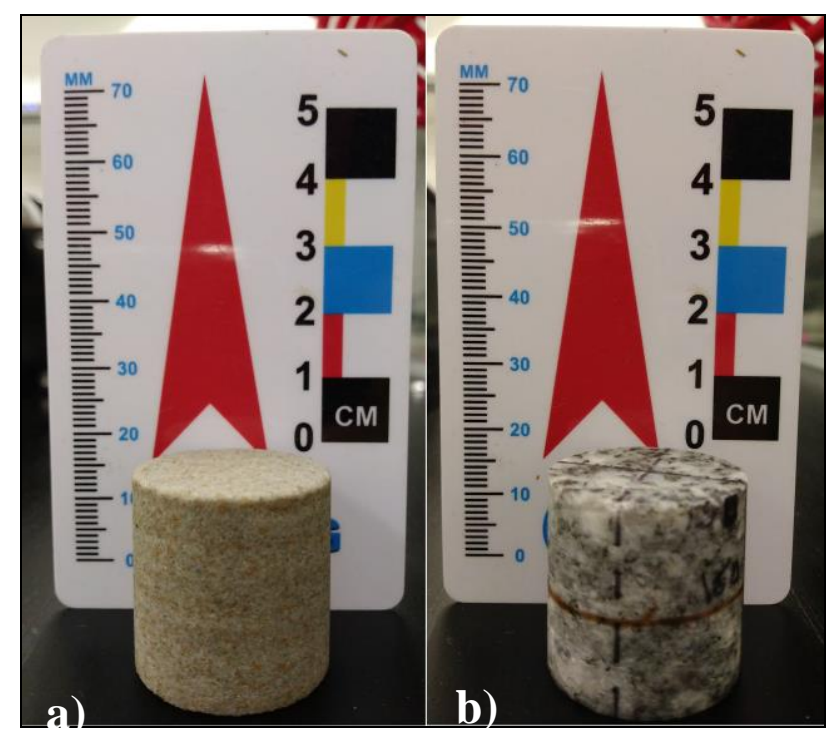

Figura 1 - Amostras utilizadas neste trabalho; a) Berea Sandstone (USA) e; b) Barre Granite (USA).

\section{Métodos}

Rochas com porosidades primária e secundária apresentaram diferentes permeabilidades, porém as 
variações das pressões e dos transientes também poderão indicar diferenças nos valores de permeabilidade.

A metodologia empregada nas medições de permeabilidade utiliza os transientes complexos tanto para rochas de alta permeabilidade como de baixa permeabilidade (Boitnott 1997). A resposta do sistema com o uso de transientes complexos é simplificada e torna-se insensível à capacidade de armazenamento da amostra, resultando em medidas mais robustas $\mathrm{e}$ confiáveis. A resposta da pressão $(P)$ para uma "função degrau em pressão de amplitude $\left(P_{0}\right)$ " em um tempo $t=0$ segue:

$$
P=P_{0}\left(1-e^{-\frac{k t}{m}}\right) \text { Eq. } 1
$$

Onde $(\mathrm{m})$ é uma constante dependente do fluido e das propriedades do sistema e da geometria da amostra; (k) é a permeabilidade. De maneira similar à perturbação senoidal, a função de transferência para esse caso em especial pode ser reescrita no domínio da frequência como:

$$
\begin{aligned}
& A_{\gamma}=\left(1+\omega^{2} m^{2} k^{2}\right) \text { Eq. } 2 \\
& \phi=-\arctan \left(\frac{k}{\omega m}\right) \text { Eq. } 3
\end{aligned}
$$

Onde $\left(A_{r}\right)$ é a razão da amplitude, $(\phi)$ é a mudança de fase e a frequência angular $(\omega)$. Para a senoidal, a permeabilidade pode ser medida utilizando ainda a razão da amplitude ou a mudança de fase.

Nesta pesquisa foram utilizados transientes complexos ASPIKE, Multipulse e SINE6.

O transiente ASPIKE possui banda larga e carrega energia de alta frequência significativa. Essa característica torna-o ideal para medidas realizadas em rocha que apresentam alta permeabilidade, mas também é aplicado em amostras de rocha com baixa permeabilidade.

De maneira semelhante ao transiente ASPIKE, o transiente Multipulse é medido quando aplicado em diferentes taxas de fluxo com tempo de duração de cada pulso dependente do tempo. É útil para realização de testes rápidos e pequeno tempo de cálculo.

O transiente SINE6 é a soma de 6 senoidais de amplitude idêntica em 6 diferentes frequências, estendendo-se como função senoidal simples. Sua limitação é decorrente de frequências mais elevadas. Como vantagem comporta-se como função "quasicontínua" e em princípio pode apresentar baixo erro, dependendo da amostra analisada (Boitnott 1997).

\section{Resultados}

A amostra do arenito da Formação Berea apresentou comportamento de alta permeabilidade submetida à Pressão Confinante $(\mathrm{Pc})$ de $30 \mathrm{MPa}$ e Pressão de Poro
(Pp) de $7 \mathrm{MPa}$. Os transientes complexos utilizados foram ASPIKE e Multipulse, com amplitude de medição em $1 \mathrm{MPa}$ e frequência de $0,5 \mathrm{~Hz}$. Os valores de permeabilidade $(\mathrm{k})$ obtidos foram $\mathrm{k}=19,6 \mathrm{mD}$ e $\mathrm{k}=18,9$ $\mathrm{mD}$, respectivamente e storage $=0,00 \%$, para ambos os transientes (Tabela 1).

Tabela 1: Arenito da Fm. Berea - Alta Permeabilidade.

\begin{tabular}{c|c|c}
\hline & Permeabilidade $(\mathrm{k})$ & Storage (\%) \\
\hline ASPIKE & $19,6 \mathrm{mD}$ & 0,0 \\
\hline Multipulse & $18,9 \mathrm{mD}$ & 0,0 \\
\hline
\end{tabular}

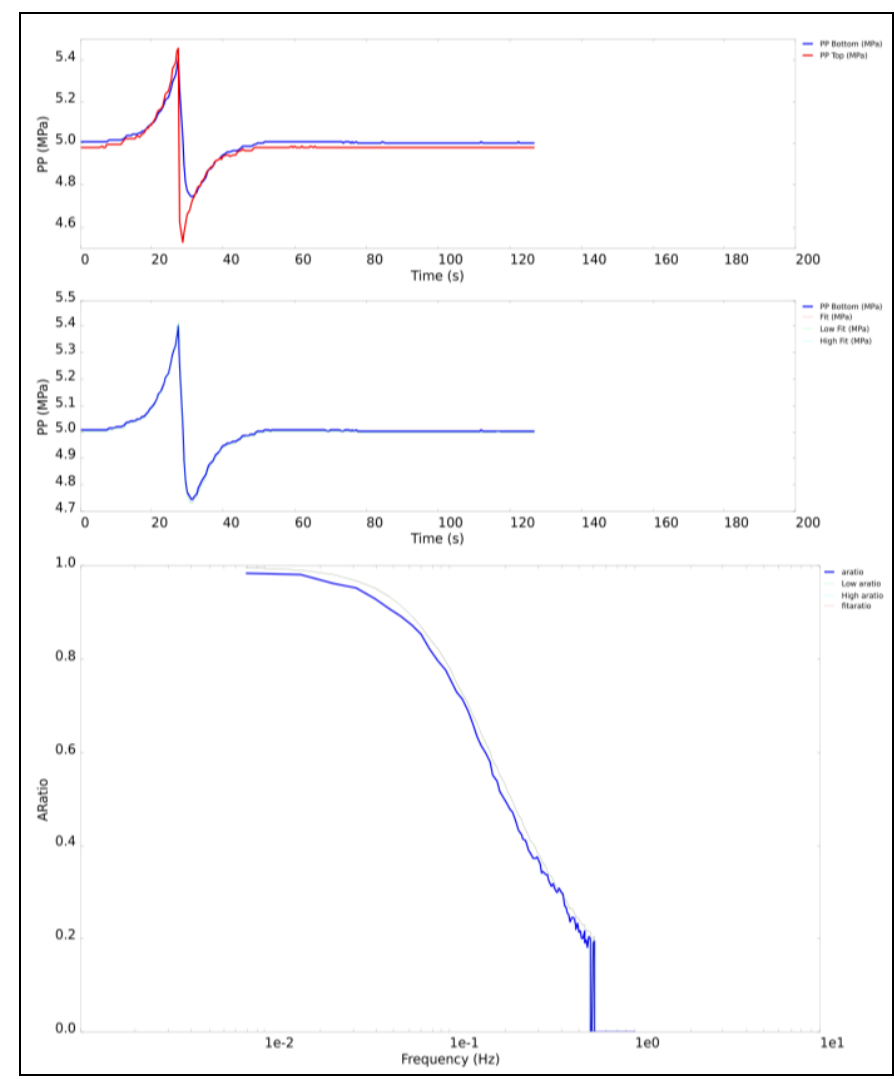

Figura 2 - Gráficos de resultados para o transiente ASPIKE do arenito $\mathrm{Fm}$. Berea, alta permeabilidade. 


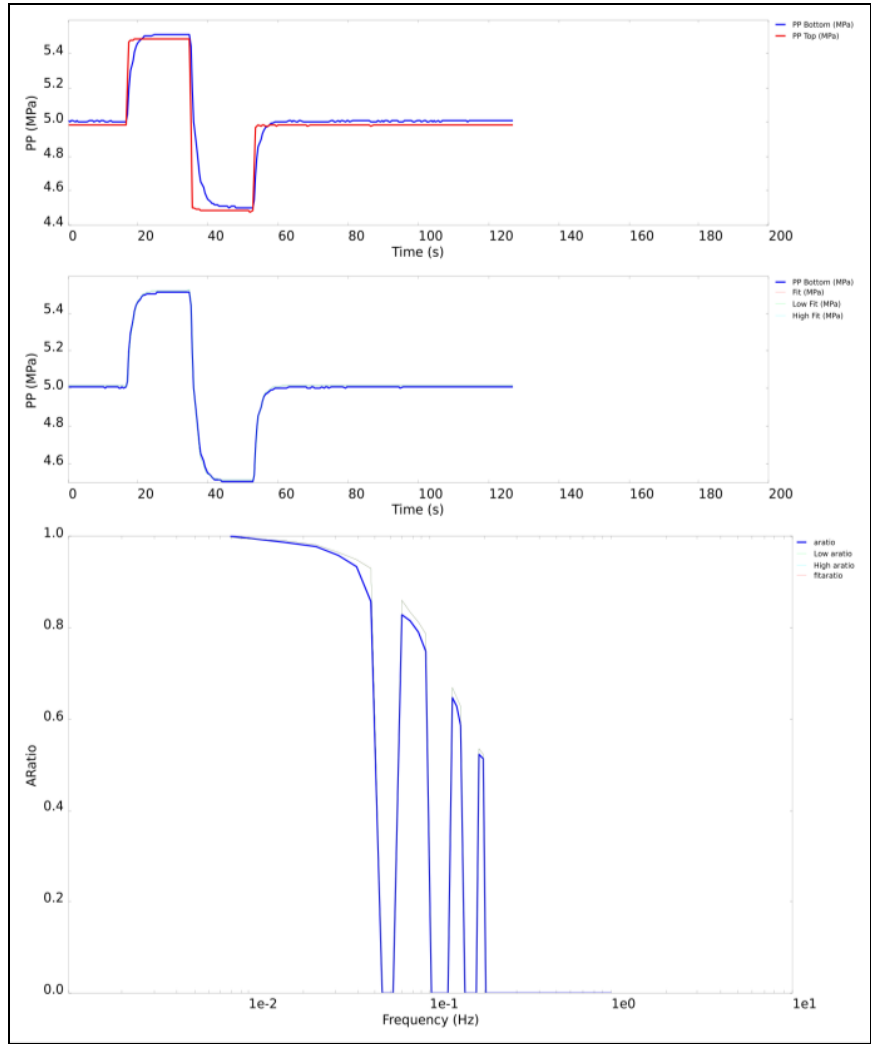

Figura 3 - Gráficos de resultados para o transiente Multipulse do arenito $\mathrm{Fm}$. Berea, alta permeabilidade.

Para o Barre Granite, foi utilizado o método de aquisição para rochas de baixa permeabilidade inicialmente com Pressão Confinante $(\mathrm{Pc})$ em $5 \mathrm{MPa}$ e Pressão de Poro $(\mathrm{Pp}) 3 \mathrm{MPa}$. Em seguida, a aquisição foi feita ao aumentar a pressão confinante para $10 \mathrm{MPa}$ e Pressão de Poro para $5 \mathrm{MPa}$. Para as medições de permeabilidade com o transiente Multipulse foi aplicado amplitude de medição em $1 \mathrm{MPa}$ e frequência de $0,5 \mathrm{~Hz}$. Os valores de permeabilidade $(\mathrm{k})$ obtidos foram de $\mathrm{k}=$ $8,38 \times 10^{-5} \mathrm{mD}$ (storage $\left.=4,242 \%\right), \mathrm{k}=10,9 \times 10^{-5} \mathrm{mD}$ (storage $=4,408 \%)$, respectivamente, conforme a tabela a seguir.

Tabela 2: Barre Granite, Baixa Permeabilidade (Multipulse).

\begin{tabular}{c|c|c|c}
\hline Faixa Pc & Faixa Pp & Permeabilidade (k) & Storage (\%) \\
\hline $5 \mathrm{MPa}$ & $3 \mathrm{MPa}$ & $8,38 \times 10^{-5} \mathrm{mD}$ & 4,242 \\
\hline $10 \mathrm{MPa}$ & $5 \mathrm{MPa}$ & $10,9 \times 10^{-5} \mathrm{mD}$ & 4,408 \\
\hline
\end{tabular}

Dessa forma, os resultados obtidos estão ilustrados nas figuras $2,3,4,5$ e 6 .

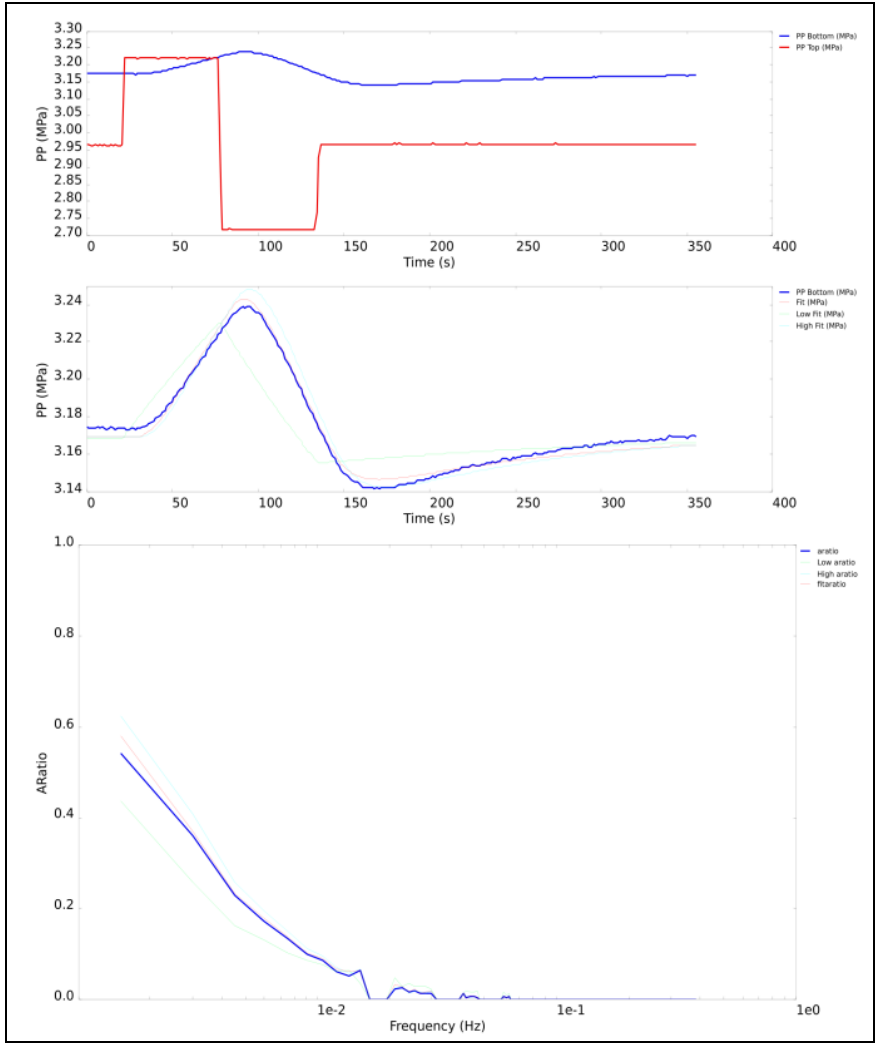

Figura 4 - Gráficos de resultados para o transiente Multipulse do Barre Granite, baixa permeabilidade, pressão confinante em 5 $\mathrm{MPa}$ e pressão de poro em $3 \mathrm{MPa}$.

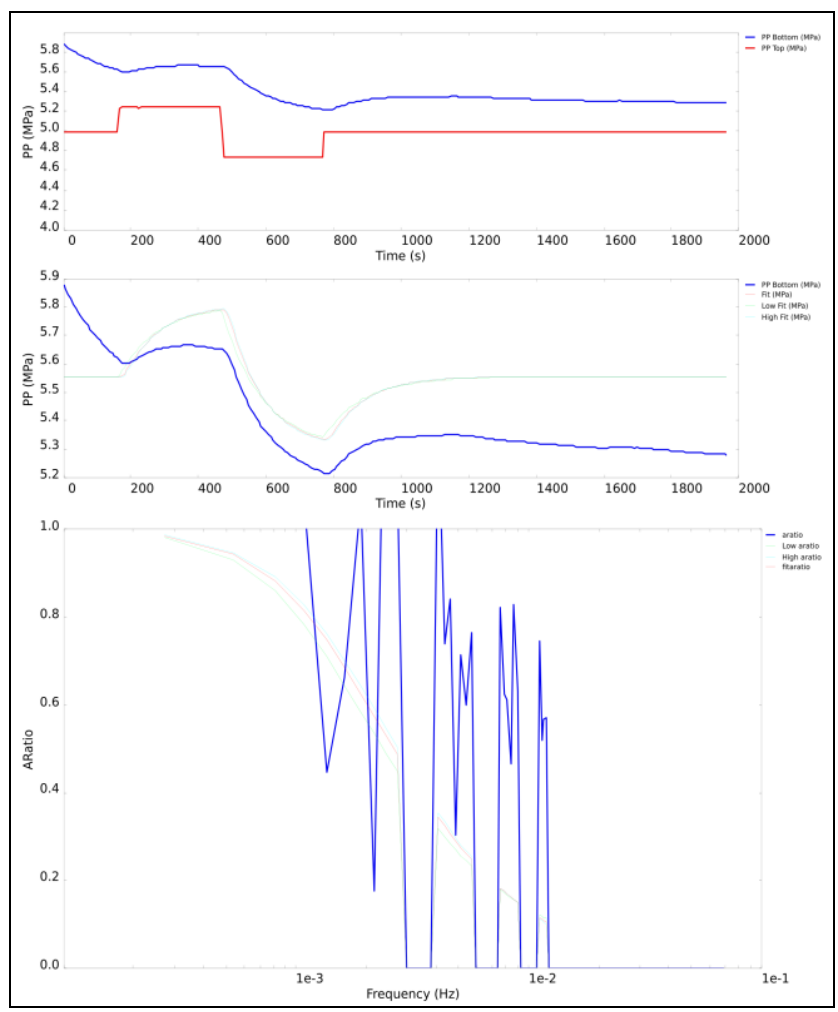

Figura 5 - Gráficos de resultados para o transiente Multipulse do Barre Granite, baixa permeabilidade, pressão confinante em 10 $\mathrm{MPa}$ e pressão de poro em $5 \mathrm{MPa}$. 


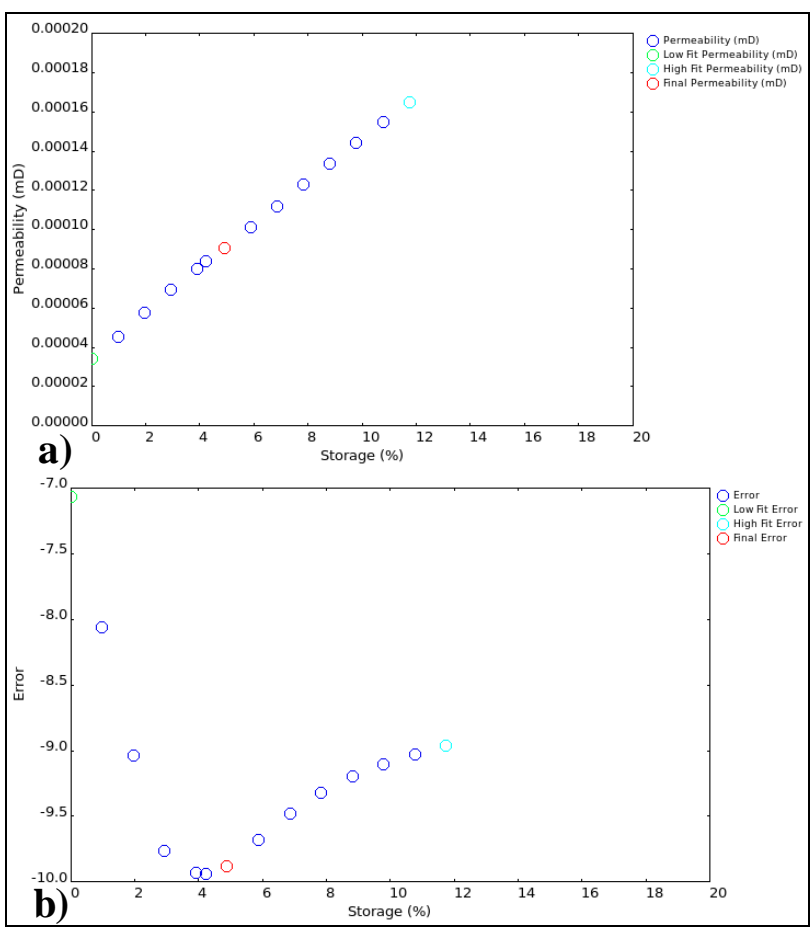

Figura 6 - a) Gráficos de Permeabilidade em função do armazenamento (Storage), acima e; b) Gráfico do Erro em função do armazenamento (Storage) para o Barre Granite, pressão confinante em $5 \mathrm{MPa}$ e pressão de poro em $3 \mathrm{MPa}$.

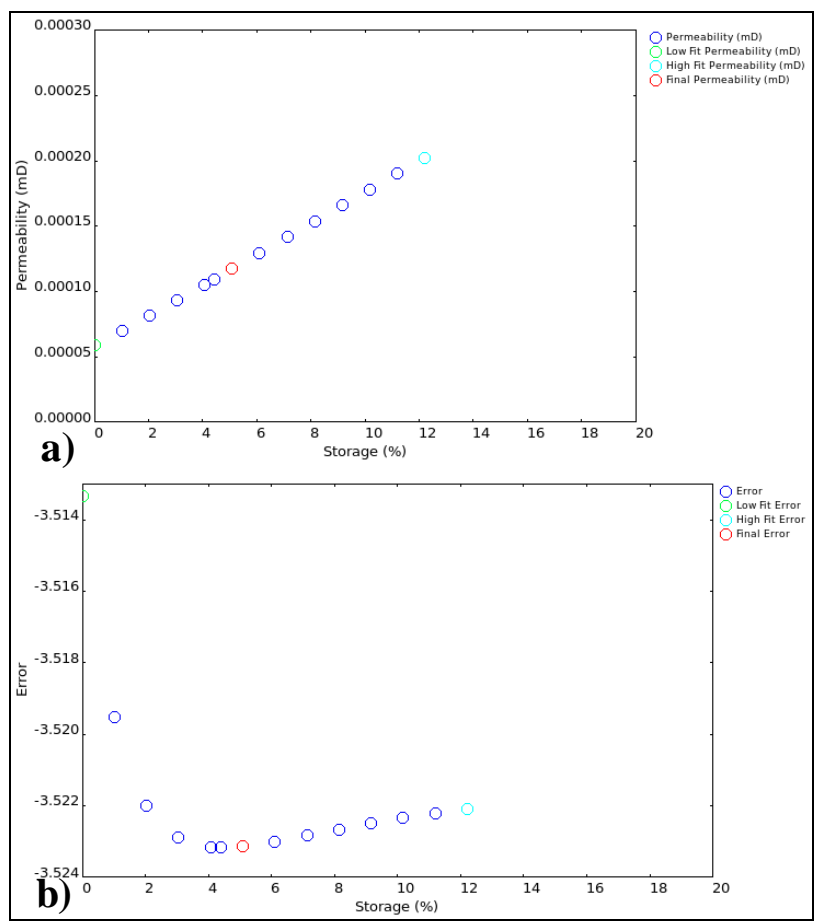

Figura 7 - a) Gráficos de Permeabilidade em função do armazenamento (Storage); e b) Gráfico do Erro em função do armazenamento (Storage). Barre Granite, pressão confinante em $10 \mathrm{MPa}$ e pressão de poro em $5 \mathrm{MPa}$.

\section{Conclusões}

Os dados obtidos atestam a eficácia dos métodos de aquisição do AutoLab 1000. A resposta de permeabilidade do arenito, Fm Berea possui alta permeabilidade, esperado para arenitos com alta porosidade, sem cimentação ou com influência de outros processos afetariam tal propriedade física. A resposta de permeabilidade baixa para o Barre Granite, também mostrou concordante com aquela esperada para rochas cristalinas que apresentam porosidade secundária, pouca ou nenhuma porosidade.

\section{Agradecimentos}

Os autores agradecem a New England Research - NER, Tennessine Instrumentação Analítica e Instituto de Geociências/UnB, pelo suporte neste trabalho.

\section{Referencências}

Boitnott, G. 1997. Use of Complex Pore Pressure Transients to Measure Permeability of Rocks. In: SPE International- Society of Petroleum Engineers., 38-45. (10.2118/38717-MS). 